\title{
PANORAMA DE CONEXIÓN DURANTE LAS CLASES VIRTUALES EN UNA MUESTRA DE ESTUDIANTES UNIVERSITARIOS
}

\section{Overview of connection on the virtual classes in a sample of university students}

\author{
Yolanda Feria-Cuevas ${ }^{1 *}$, Marisela Rodríguez-Morán ${ }^{3}$, Martha Isabel Torres-Morán² y \\ Enrique Pimienta-Barrios ${ }^{2}$
}

\begin{abstract}
${ }^{1}$ Departamento de Ciencias Ambientales. ${ }^{2}$ Departamento de Producción Agrícola. Centro Universitario de Ciencias Biológicas y Agropecuarias. Camino Ing. Ramón Padilla Sánchez No. 2100, Predio las Agujas, Zapopan, Jalisco 45200. ${ }^{3}$ Depto. de Teorías e Historias. Centro Universitario de Arquitectura Arte y Diseño. Calzada Independencia Norte No. 5075, Huentitán el Bajo S.H. 44250.
\end{abstract}

Autor para la correspondencia: yolanda.feria@academicos.udg.mx

\section{Resumen}

El presente artículo es una recopilación de información obtenida directamente de un grupo de alumnos y profesores de dos Centros Universitarios pertenecientes a la Red de la Universidad de Guadalajara. Se pretende brindar un panorama de desarrollo de las clases virtuales durante la segunda parte del ciclo escolar 2020-A (enero-junio 2020) en el cual intempestivamente, tanto alumnos como profesores, se vieron obligados a trabajar desde el confinamiento debido al riesgo por la pandemia por COVID-19. Se encontró que un alto porcentaje (del 68 al $84 \%$ ) de alumnos y maestros en la muestra estudiada, a pesar del ambiente de incertidumbre, trabajaron en conjunto para llevar a buen término las materias que se iniciaron en forma presencial. Así mismo, los datos obtenidos reflejan que es un porcentaje muy bajo el alumnado que no cuenta con un medio para atender los temas que se refieren a cada materia y reportaron conocer diversas plataformas por las cuales los profesores pueden darle seguimiento a las clases. Sin duda, el reto es mejorar las condiciones del ambiente enseñanza- aprendizaje en los tiempos modernos e implementar estrategias que mejoren el ambiente de conectividad, explorando desde la perspectiva institucional, mejores oportunidades de capacitación a toda la red universitaria.

Palabras clave: Conectividad estudiantil, enseñanza virtual, aprender en la pandemia
Abstract
This paper is a compilation of information obtained directly from a group of students and teachers from two University Centers belonging to the University of Guadalajara. The aim is to provide an overview of the development of virtual classes during the second part of the 2020-A school year (January-June 2020), in which, unexpectedly, both students and teachers were forced to work from confinement due to the risk of COVID-19 pandemic. It was found that a high percentage (from 68 to $84 \%$ ) of students and teachers in the studied 
sample, despite the uncertain environment, worked together to successfully complete the subjects that were started as classroom learning. Likewise, the data obtained reflects that it is a very low percentage of the students who do not have the possibility to attend to the topics that refer to each subject and reported knowing various platforms through which teachers can follow up on classes. Undoubtedly, the challenge is to improve the conditions of the teachinglearning environment in modern times and implement strategies that improve the connectivity environment, exploring, from an institutional perspective, better training opportunities for the entire university network.

Key words: Student connectivity, virtual teaching, learning in the pandemic

\section{Introducción}

Nuevamente la humanidad se enfrenta a una realidad inesperada, la aparición, propagación e impacto de la enfermedad llamada COVID-19, causada por el virus SARS-CoV-2 que tomó por sorpresa al mundo, interrumpiendo las actividades cotidianas de la humanidad en todos sus ordenes. Las medidas de seguridad social que se están tomando para controlar el impacto de la enfermedad, obligaron a un confinamiento inmediato que ha desestabilizado a las sociedades y sus actividades, y de inicio creó incertidumbre. En México, el primer caso de esta enfermedad, fue reportado el día 28 de febrero y el 11 de marzo la organización Mundial de la Salud emitió una declaratoria de pandemia (OMS, 2020). Ante esta situación, las Instituciones de educación tuvieron que hacer frente e implementar estrategias y acciones a corto plazo, en donde todos los actores se debieron unir para llevar a buen término el que pudiera ser llamado "experimento de trabajar y aprender en casa" (Toledo-Ibarra et al., 2020). Si bien se ha manejado desde hace tiempo la necesidad del uso de tecnologías de la información y comunicación (TIC) para apoyar el proceso enseñanza-aprendizaje y adaptar la relación educador-educandos, la realidad de nuestro país es tan compleja, que rebasa los modelos diseñados para una realidad estática que no permitió la adopción de herramientas necesarias para adaptarse a un entorno dinámico, global y de interacciones constantes (Palma, 2016). El hecho es que en estos meses en que se tuvo que adoptar un modelo emergente para llevar las clases de forma virtual, se tiene presente las inconveniencias que puede representar para la población estudiantil que se vio obligada a regresar a sus comunidades que en ocasiones presentan limitaciones de las fuentes que suministran internet o simplemente no cuentan con lo básico para interactuar en línea o de forma virtual.

En el presente trabajo-se presenta estadística relativa a la condición de conectividad de una muestra de alumnos del Centro Universitario de Ciencias Biológicas y Agropecuarias (CUCBA) y del Centro Universitario de Arte Arquitectura y Diseño (CUAAD), con el objetivo de documentar a partir de una pequeña muestra, el panorama de lo acontecido durante los meses de marzo a mayo, tiempo en el que se implementaron las clases virtuales.

\section{Materiales y métodos}

Se realizaron encuestas electrónicas a través de formulario Google. Las preguntas integradas en el formulario se 
muestran en el Cuadro1. El total de alumnos encuestados fue de 352 alumnos con status de regular de las carreras de Ingeniero Agrónomo (Agronomía), Licenciado en Agronegocios y Licenciada en Biología (CUCBA) y de Diseño industrial, Diseño de la comunicación gráfica y Diseño de interiores y ambientación (CUAAD). Las preguntas fueron relativas a su forma y estado de conectividad, así como al número de materias que atendieron durante el ciclo escolar 2020A y cuáles de ellas se continuaron en forma virtual al comenzar el confinamiento y hasta el final del ciclo escolar. Además, fueron encuestados un grupo de profesoras y profesores con respecto al número de alumnos en su lista al inicio del semestre (un total de 474 alumnos) y cuántos de ellos continuaron la las actividades en forma virtual. Se utilizó estadística descriptiva para obtención de media y moda. Los resultados se presentan a través de Cuadros y gráficas.

Cuadro 1. Reactivos en el formulario

1. Sexo

2. ¿En qué Centro Universitario estudias?

3. ¿Qué carrera estudias?

4. ¿Tienes computadora? [De escritorio]

5. ¿Tienes computadora? [Portátil]

6. ¿Tienes teléfono celular?

7. ¿En dónde te conectas a Internet?

8. ¿Cuáles son las plataformas que utilizaron tus profesores para las clases en línea?

9. ¿Crees que tus profesores requieren capacitación para impartir clases en línea?

10. ¿Crees que tú requieres capacitación para recibir clases en línea?

11. ¿Cuántas materias tenías de manera presencial este semestre?

12. ¿Cuántas materias tienes en línea este semestre?

\section{Resultados y discusión}

La distribución de las respuestas por sexo y Centro Universitario, se presenta en el Cuadro 2.

Cuadro 2. Distribución de los encuestados, por sexo y Centro Universitario

\begin{tabular}{|c|c|c|}
\hline Centro universitario & Mujer & Hombre \\
\hline CUCBA & 121 & 115 \\
\hline CUAAD & 87 & 29 \\
\hline TOTAL & 208 & 144 \\
\hline
\end{tabular}

¿Cómo se conectan a las clases virtuales?

Con respecto a la posibilidad de conectarse a sus clases virtuales, en el Cuadro 3 se muestra el resultado de la encuesta con los porcentajes que representan la conectividad de los alumnos de cada carrera. De los datos presentados, se puede concluir que en su mayoría, los alumnos cuentan con los medios para conectarse a las clases virtuales, que si bien significa un estatus económico que no todos poseen, el porcentaje de alumnos con estas posibilidades, es alentador. Trabajar 
desde casa, significa enfrentarse a muchos retos, poseer un lugar confortable, suficiente espacio, alejarse del ruido y tener conectividad (UNESCO, 2020).

Cuadro 3. Alumnos con herramientas de conexión a clases virtuales y porcentaje (\%) con respecto al total de los alumnos encuestados.

\begin{tabular}{|c|c|c|c|c|c|}
\hline Carrera & $\begin{array}{c}\text { alumn@s } \\
\text { total }\end{array}$ & $\begin{array}{c}\text { con } \\
\text { celular }\end{array}$ & $\begin{array}{c}\text { con } \\
\text { internet }\end{array}$ & $\begin{array}{c}\text { con } \\
\text { computadora } \\
\text { de escritorio }\end{array}$ & $\begin{array}{c}\text { con } \\
\text { computadora } \\
\text { portátil }\end{array}$ \\
\hline Ingeniero Agrónomo & 133 & $128(96)$ & $110(83)$ & $38(29)$ & $93(70)$ \\
\hline Agronegocios & 67 & $67(100)$ & $63(94)$ & $19(28)$ & $44(66)$ \\
\hline Biología & 37 & $37(100$ & $32(86)$ & $5(14)$ & $24(65)$ \\
\hline Diseño Industrial & 46 & $46(100)$ & $41(89)$ & $19(41)$ & $36(78)$ \\
\hline Diseño de Interiores y ambientación & 35 & $35(100)$ & $29(83)$ & $8(8)$ & $25(71)$ \\
\hline Diseño para la comunicación gráfica & 33 & $33(100)$ & $33(100)$ & $6(18)$ & $29(88)$ \\
\hline
\end{tabular}

Los estudiantes han tenido que hacer un esfuerzo para adaptarse a lo que para muchos de ellos son nuevas fórmulas para aprender. En el caso de los estudiantes encuestados en el presente trabajo, los resultados prueban que han podido encontrar una oferta de continuidad. En la Figura 1, se muestra el porcentaje de cursos virtuales que mencionaron los alumnos, en contraste con las que deberían llevar en el semestre. Cabe mencionar que la totalidad de materias fueron evaluadas, por lo que incluso los profesores que no impartieron cursos en línea tuvieron que evaluar el curso y dar el seguimiento utilizando algún medio electrónico para recibir el trabajo final de la materia.

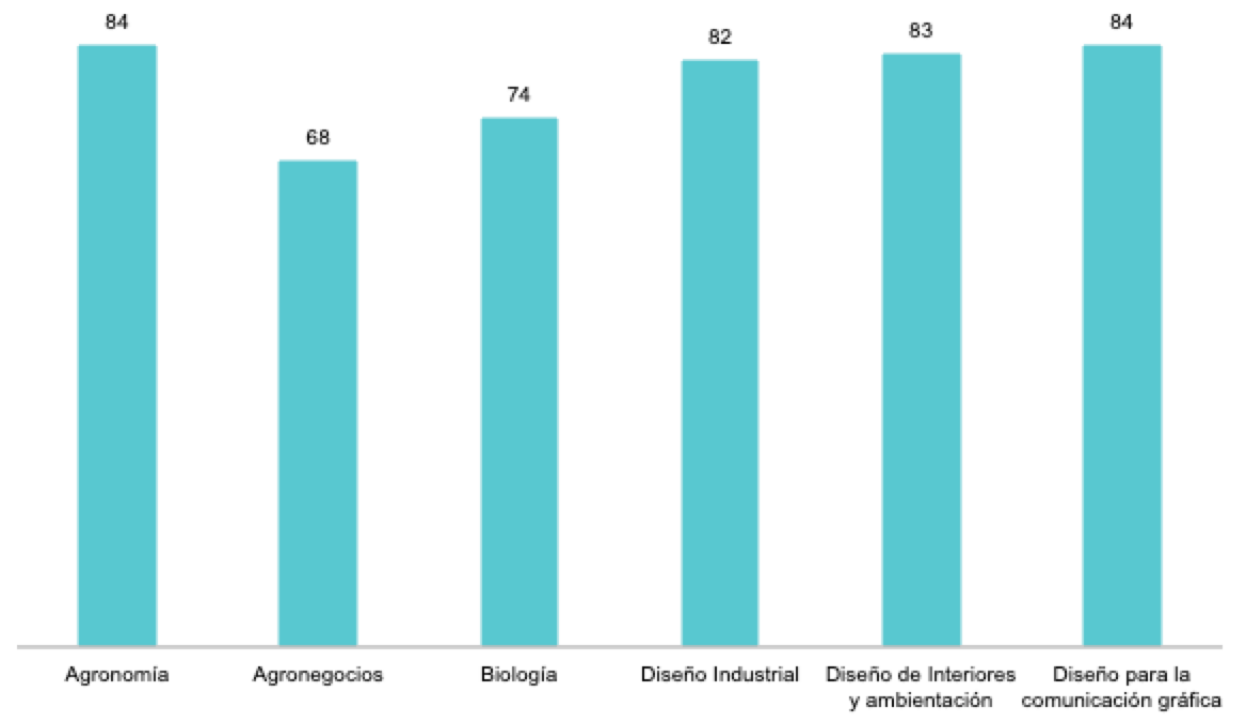

Figura 1. Porcentaje de cursos del semestre que tuvieron continuidad en línea 
Según la UNESCO (2020), la conectividad se está extendiendo a nivel mundial, aún cuando la realidad es de baja conectividad en hogares de países de bajos y medianos ingresos. En el caso de América Latina y el Caribe, reporta que solo uno de cada dos hogares está conectado. En el caso del presente trabajo, se encontró un nivel de conectividad del 83 al $100 \%$ dividido según la carrera de la que se trate (Cuadro $3)$.

Plataformas en línea utilizadas por carrera

Para que las clases en línea sean aprovechadas al máximo por los alumnos, es necesario que los profesores mantengan el interés de los estudiantes y los motiven a participar y realizar las actividades planteadas, ofreciendo diversos recursos para la obtención de información y conservando la interacción profesor-alumno, que permita resolver dudas y sentir acompañamiento en el proceso de aprendizaje (Organista y Backhoff, 2002; Castro et al., 2007; González y Martínez, 2013; Jimoyiannis y Tsiotakis 2019). En las Figura 2 y 3, se muestran las plataformas que los profesores ofrecieron como modo de conexión con sus alumnos, lo cual ofreció diversidad y versatilidad.

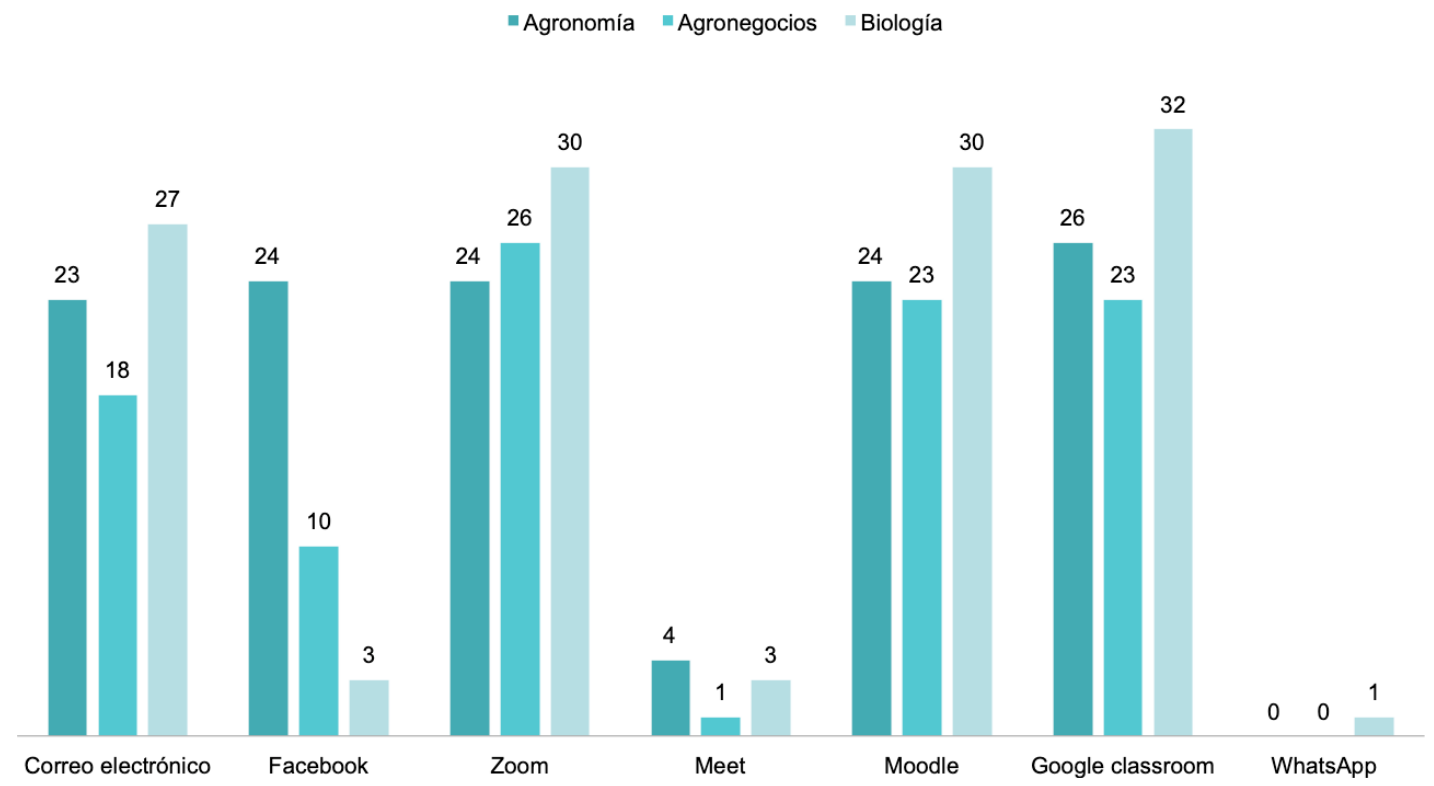

Figura 2. Plataformas utilizadas para los cursos virtuales en el CUCBA 


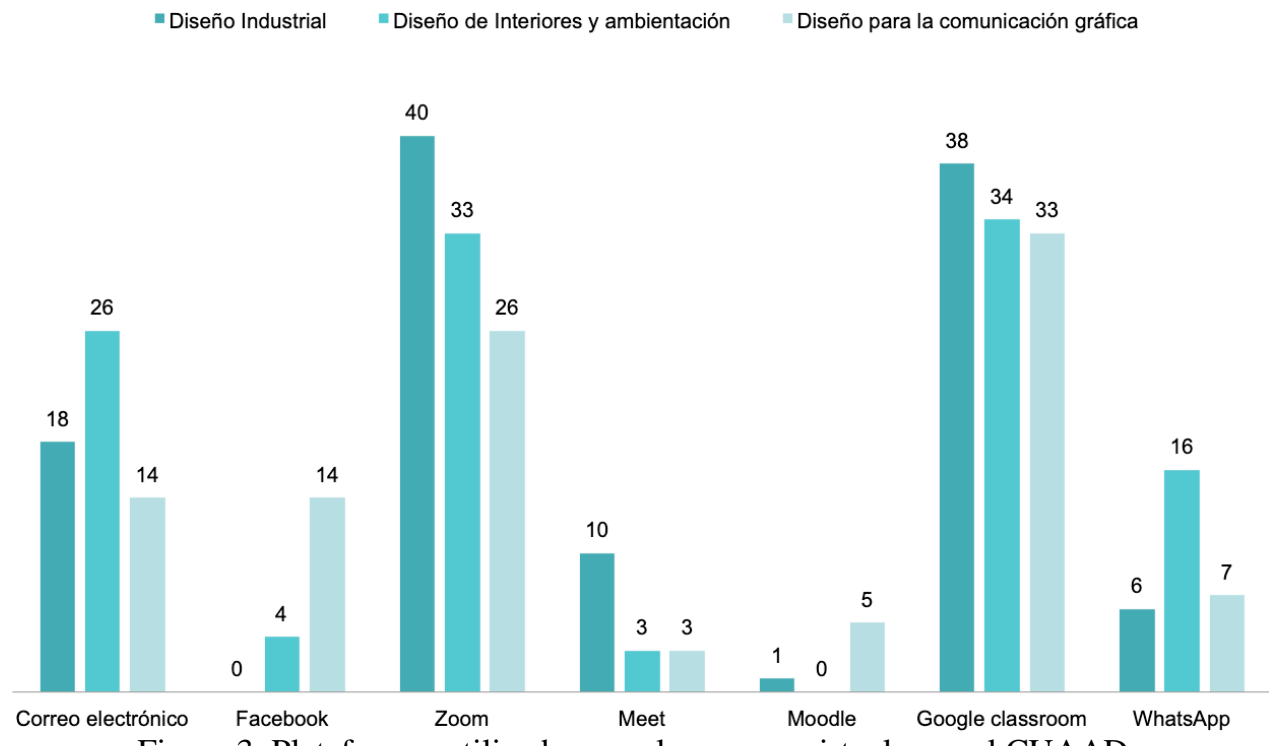

Figura 3. Plataformas utilizadas para los cursos virtuales en el CUAAD

El desarrollo de las tecnologías de la información y la comunicación (TIC), desde su masificación hace 30 años, ha cambiado la dinámica de las interacciones personales. Este desarrollo ha impactado en todos los sectores de la vida cotidiana, sin embargo, tal como lo mencionan González y Martínez (2013), en el sector educativo la incorporación de las TIC, ha sido más lenta ya que implica romper paradigmas arraigados en la educación formal, que ha sido preferentemente presencial. En la situación del entorno de este trabajo, el uso de esta tecnología, abrió el panorama para la integración de recursos y procesos para apoyar la enseñanza y el aprendizaje. Se tiene por cierto, que el uso de las TIC, promueve el incremento de habilidades en los alumnos, tales como la búsqueda y análisis de la información, redacción, concreción de ideas y consolidación de la interacción alumno profesor (Castro et al., 2007).

El proceso de clases virtuales no debería ser visto desde la sola perspectiva de los alumnos, ya que ha tenido un impacto muy significativo entre los profesores que se han visto en la necesidad de acceder al uso de diferentes plataformas y adaptar sus condiciones personales al desarrollo de dicha actividad, al igual que los estudiantes, mismos que reportan la necesidad de capacitación (Figura 4). 


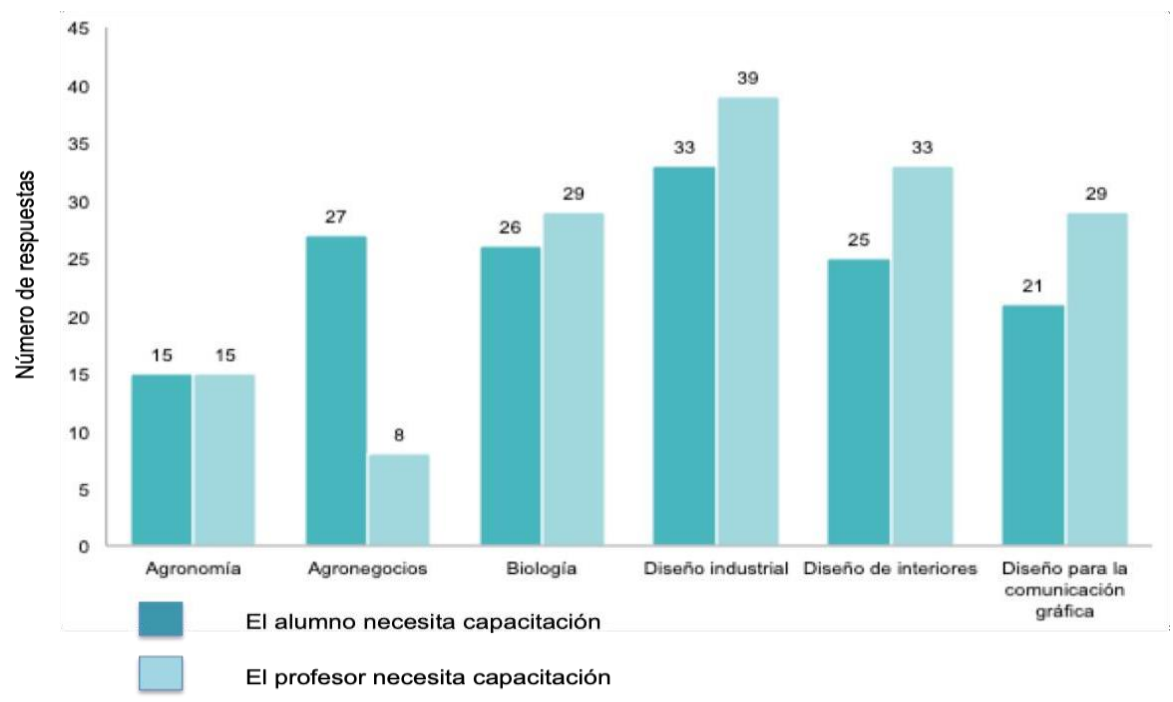

Figura 4. Necesidad de capacitación que identificaron los encuestados, para el alumno o para el profesor

De los datos proporcionados por los profesores, destaca el número de alumnos inscritos a su materia, los que no asistieron desde el inicio del semestre y los que continuaron con las clases en línea. Como puede observarse en la Figura 5, de los 474 alumnos contabilizados en las listas de los maestros encuestados, el 14\% (68) no se presentaron a clases, y en el caso del presente muestreo, de la cantidad de alumnos que asistían de manera regular a las clases presenciales, sólo el 5.2\% no atendieron las clases virtuales (Figura 5).

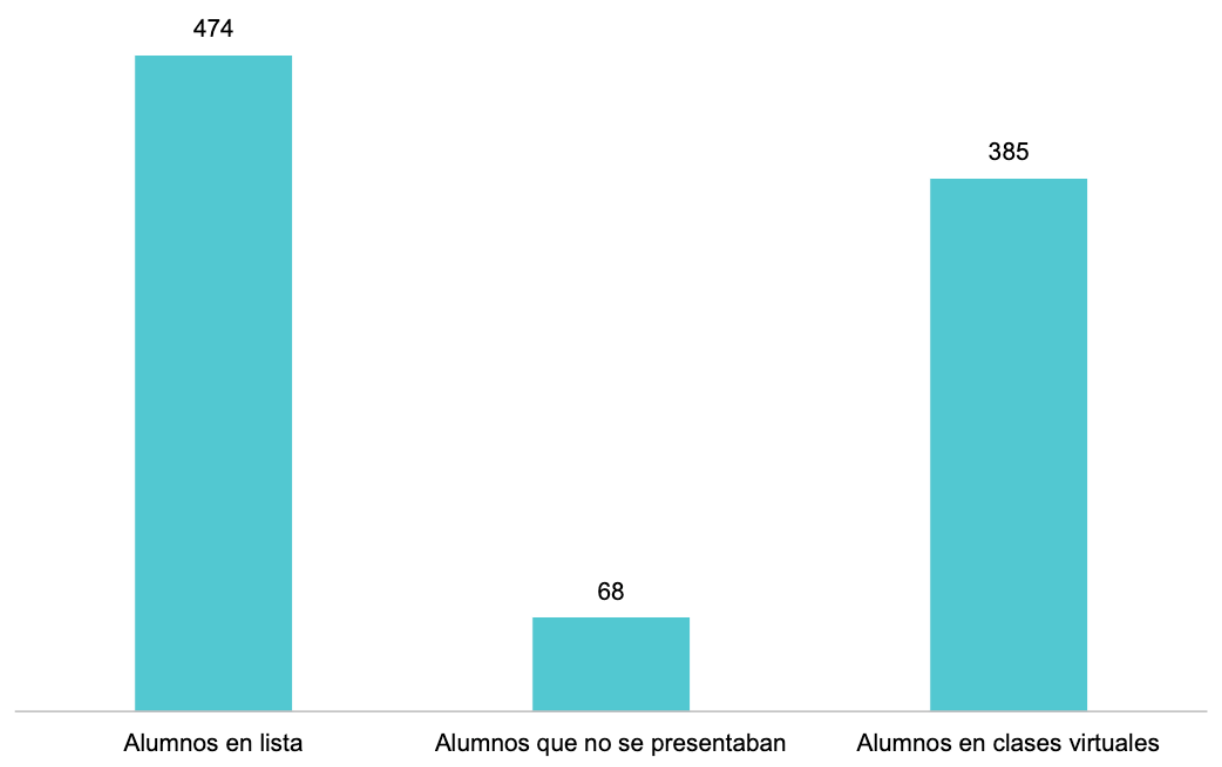

Figura 5. Continuidad de asistencia de los alumnos durante las clases virtuales 


\section{Conclusiones}

De los resultados obtenidos, se concluye que si bien, el impacto de la pandemia de Covid - 19 en las Instituciones de Educación Superior fue inesperado y en la mayoría de los casos no había otro plan de contingencia que no sea intentar continuar con las clases en forma virtual o remota, para el caso de los centros universitarios analizados, se observó que es necesario homogeneizar en lo posible, el uso de herramientas tecnológicas tanto para los profesores como para los alumnos. Implementar políticas para minimizar la desarticulación entre la educación básica y la superior y brindar la oportunidad y acceso a la educación a un mayor número de alumnos. Esto aseguraría una salida ante este tipo de crisis, con el mayor grado posible de inclusión y equidad, tal como lo recomienda la UNESCO. De hecho, la situación ante esta pandemia, agregó un mayor grado de complejidad a la educación superior a nivel mundial. Particularmente en nuestra Universidad, surge la necesidad de evaluar el impacto que este proceso tuvo en la calidad de la enseñanza, así como medir la eficacia de las herramientas utilizadas y que esto conlleve a diseñar e implementar una política de procesos para la educación virtual en los centros universitarios.

\section{Literatura citada}

Castro, S., Guzmán, B. y Casado, D. (2007). Las Tic en los procesos de enseñanza y aprendizaje Laurus, 13(23):213-234
González, A. G. P. y Martínez, F. P. (2013). Algunas reflexiones en torno al proceso de transición del docente presencial al docente elearning. Ponencia presentada en el 7th International Technology, Educationand Development Conference. Recuperado de https://educatic.unam.mx/publica ciones/ponencias/4- reflexiones.pdf 8 de junio 2020

Jimoyiannis A., Tsiotakis P. (2019) Students' Engagement and Peer Interaction in On-Line Academic Writing Through a Course Blog. In: Tsitouridou M., A. Diniz J., Mikropoulos T. (eds) Technology and Innovation in Learning, Teaching and Education. TECHEDU 2018. Communications in Computer and Information Science, vol 993. Springer, Cham

OMS, (2020) Declaración conjunta de la ICC y la OMS: Un llamamiento a la acción sin precedentes dirigido al sector privado para hacer frente a 1 COVID-19.

https://www.who.int/es/newsroom/detail/16-03-2020-icc-whojoint-statement-an-unprecedentedprivate-sector-call-to-action-totackle-covid-19 (página consultada el 30 de mayo 2020)

Organista, S. J. y Backhoff, E. E. (2002). Opinión de estudiantes sobre el uso de apoyos didácticos en línea en un curso universitario. Revista electrónica de investigación educativa 4(1)

Palma, P.V. (2016). Edupunk: el sistema educativo que México necesita. Revista Forbes. Junio 11.

Toledo-Ibarra et al., (2020). The COVID19 pandemic from the 
YOLANDA FERIA-CUEVAS ET AL

multidisciplinary visión of 28 university professors of Nayarit

Mexico. Revista Biociencias 7-e976.

DOI://doi.org

10.15741/revbio.07.e976

UNESCO, (2020). COVID-19 and higher education: Today and tomorrow. Impact, analysis, policy responses and recommendations. IESALC. pp: 46. 